\title{
LOCALLY-FINITELY-VALUED COHOMOLOGY GROUPS
}

\section{WILLIAM L. GORDON}

1. Introduction. It is known that for a compact Hausdorff $\left(T_{2}\right)$ space, the finitely valued functions [3] are sufficient to determine the Alexander-Kolmogoroff groups of the space. In this note we generalize this result by showing that for a fully normal $T_{2}$ space the cochains which are locally finitely-valued are sufficient to determine the theory. This result, together with the isomorphisms established by Dowker [1] and Yang [7], shows that the groups defined below agree with those of the Cech theory based on arbitrary coverings, at least on the class of fully normal spaces. The last section concerns a variation adapted for the locally-compact case.

2. The cohomology groups. In this section we shall again define the Alexander-Kolmogoroff groups, cf. Spanier [4], giving parallel development to the finitely-valued groups considered by Keesee [3] and the locally-finitely-valued groups of interest here.

For a given topological space $X$ and a (fixed, but arbitrary) abelian group $G$ the basic group $C^{p}(X)$ of $p$-cochains is the group of all functions on $X^{p+1}$ (the cartesian product of $X$ with itself $p+1$ times) with values in $G$, the group operation being defined by pointwise addition. The coboundary homomorphism $\delta$ defined by, for $\phi \in C^{p}(X)$ and $\left(x_{0}, \cdots, x_{p+1}\right) \in X^{p+2}$,

$$
\delta \phi\left(x_{0}, x_{1}, \cdots, x_{p+1}\right)=\sum_{i=0}^{p+1}(-1)^{i} \phi\left(x_{0}, \cdots, x_{i-1}, x_{i+1}, \cdots, x_{p+1}\right)
$$

has the fundamental property that $\delta \delta=0$.

For any collection $U$ of subsets of $X$ write $U^{(p+1)}=U\left\{U^{p+1} \mid U \in V\right\}$. In particular if $U$ is an open covering of $X$, then $U^{(p+1)}$ is a neighborhood of the diagonal of $X^{p+1}$. Conversely, any neighborhood of the diagonal of $X^{p+1}$ contains $U^{(p+1)}$ for some open covering $U$ of $X$. For any subset $Q$ of $X^{p+1}$ and $\phi \in C^{p}(X)$ denote by $\phi \mid Q$ the function $\phi$ regarded as defined only on $Q$. With the above notations in mind, for a subset $A$ of $X$, define a subgroup $C^{p}(X, A)$ of $C^{p}(X)$ to be $\left\{\phi \in C^{p}(X) \mid \exists\right.$ an open covering $\bigcup$ of $\left.A \cdot \ni \cdot \phi \mid \bigcup^{(p+1)} \cap A^{p+1}=0\right\}$, the subgroup of $p$-cochains locally zero on $A$. The auxiliary notation

Presented to the Society November 29, 1952 under the title On the coefficient group in cohomology; received by the editors October 23, 1954. 
$C_{0}^{p}(X)$ will frequently be used instead of $C^{p}(X, X)$. Clearly, for any $A, C_{0}^{p}(X) \subset C^{p}(X, A)$ and $\delta C^{p}(X, A) \subset C^{p+1}(X, A)$.

We shall be concerned with the subgroup $L^{p}(X) \subset C^{p}(X)$ of those $p$-cochains which locally are finite valued, i.e., $\phi \in C^{p}(X)$ will belong to $L^{p}(X)$ provided for each $y=\left(x_{0}, \cdots, x_{p}\right) \in X^{p+1}$, there is a neighborhood $P$ of $y$, open in the product topology, with the property that $\phi \mid P$ takes on only a finite number of values in $G$. Similarly $F^{p}(X)$ will denote the subgroup of $C^{p}(X)$ consisting of those $p$-cochains having only a finite number of members of $G$ as values. Clearly $F^{p}(X)$ $C L^{p}(X) \subset C^{p}(X)$ and it is easy to verify that $\delta F^{p}(X) \subset F^{p+1}(X)$ and $\delta L^{p}(X) \subset L^{p+1}(X)$. Therefore, defining $L^{p}(X, A)=L^{p}(X) \cap C^{p}(X, A)$, with $L_{0}^{p}(X), F^{p}(X, A)$, and $F_{0}^{p}(X)$ defined as the intersections of the appropriate subgroups, we have $F^{p}(X, A) \subset L^{p}(X, A) \subset C^{p}(X, A)$ and $\delta L^{p}(X, A) \subset L^{p+1}(X, A)$, etc.

Defining $Z^{p}(X, A)=\delta^{-1} C_{0}^{p+1}(X) \cap C^{p}(X, A), Z_{L}^{p}(X, A)=\delta^{-1} L_{0}^{p+1}(X)$ $\cap L^{p}(X, A)$ and $Z_{F}^{p}(X, A)=\delta^{-1} F_{0}^{p+1}(X) \cap F^{p}(X, A)$ as the group of $p$-cocycles of $X \bmod A$, the group of locally-finitely-valued $p$-cocycles of $X \bmod A$, and finitely-valued $p$-cocycles of $X \bmod A$ respectively (all with coefficients in $G$ ) we have $Z_{P}^{p}(X, A) \subset Z_{L}^{p}(X, A) \subset Z^{p}(X, A)$. Furthermore, defining, for $p>0, B^{p}(X, A)=\delta C^{p-1}(X, A)+C_{0}^{p}(X)$, $B_{L}^{p}(X, A)=\delta L^{p-1}(X, A)+L_{0}^{p}(X)$, and $B_{F}^{p}(X, A)=\delta F^{p-1}(X, A)+F_{0}^{p}(X)$ as the groups of $p$-coboundaries of $X \bmod A$, locally-finitely-valued $p$-coboundaries, and finitely-valued $p$-coboundaries respectively, we have (with $B^{0}(X, A)=B_{L}^{0}(X, A)=B_{F}^{0}(X, A)=0$ ) the inclusions $B_{F}^{p}(X, A) \subset B_{L}^{p}(X, A) \subset B^{p}(X, A)$ as well as the fundamental relations

$$
B_{F}^{p}(X, A) \subset Z_{F}^{p}(X, A), B_{L}^{p}(X, A) \subset Z_{L}^{p}(X, A) \text {, and } B^{p}(X, A) \subset Z^{p}(X, A) \text {. }
$$

Hence, defining the quotient groups $H^{p}(X, A)=Z^{p}(X, A) / B^{p}(X, A)$, $H_{L}^{p}(X, A)=Z_{L}^{p}(X, A) / B_{L}^{p}(X, A)$, and $H_{F}^{p}(X, A)=Z_{F}^{p}(X, A) / B_{F}^{p}(X, A)$ as the $p$ th Alexander-Kolmogoroff cohomology group of $X \bmod A$ with coefficients in $G$, the $p$ th locally-finitely-valued cohomology group, and the $p$ th finitely valued cohomology group respectively, we have that the inclusion homomorphisms $\sigma: Z_{F}^{p}(X, A)$ $\subset Z_{L}^{p}(X, A), \rho: Z_{L}^{p}(X, A) \subset Z^{p}(X, A)$, and $\tau=\rho \sigma: Z_{F}^{p}(X, A) \subset Z^{p}(X, A)$ behave in like manner on the subgroups of $p$-coboundaries. Hence by the so-called Induced Homomorphism Theorem (cf. EilenbergSteenrod [2, pp. 7-8]), there are induced homomorphisms $\rho^{*}: H_{L}^{p}(X$, $A) \rightarrow H^{p}(X, A)$ and $\tau^{*}: H_{P}^{p}(X, A) \rightarrow H^{p}(X, A)$ which furthermore are natural in the sense that they commute with the coboundary operator and the homomorphisms induced by continuous functions, these being defined for the groups introduced here by restricting the usual 
definitions (cf. Spanier [4]) given in terms of the cochain group $C^{p}(X)$ to the groups $L^{p}(X)$ and $F^{p}(X)$ respectively.

The latter homomorphism $\tau^{*}$ has been examined by Keesee [3] in the case that $X$ is compact $T_{2}$ and $A$ is closed; in which case $\tau^{*}$ is an isomorphism onto, thereby establishing that the finitely-valued cochains give rise, on the class of compact closed pairs, to a cohomology theory isomorphic to the Alexander-Kolmogoroff theory and hence to the (unique) theory satisfying the Eilenberg-Steenrod axioms (including the continuity axiom). It is our intention here to examine the homomorphism $\rho^{*}$ with the view toward a like result on a larger class of spaces.

3. Fully normal spaces. Let $U$ and $V$ be open coverings of a space $X$. We say that $V$ refines $V$ if every member of $V$ is contained in some member of $\mathcal{V}$. For each $U \in \mathcal{V}$, the star of $U=\operatorname{st}(U)$ defined as $U\left\{U^{\prime} \in U \mid U^{\prime} \cap U\right.$ is not void $\}$ is an open set. The covering $U$ starrefines $\mathcal{V}$ if $\{\operatorname{st}(U) \mid U \in U\}$ refines $\nu$, and a topological space is said to be fully normal (Tukey [6]) if every open covering has a starrefinement. Among fully normal spaces are included compact $T_{2}$ spaces (supra, compact normal spaces), metric spaces, and locally compact $T_{2}$ topological groups.

A covering $U$ is said to be locally-finite provided for each point $x \in X$ there is a neighborhood $P$ of $x$ which has a nonvoid intersection with only a finite number of members of $U$. A space is paracompact if every open covering possesses a refinement which is locally-finite.

The basic result in this regard is a theorem of A. H. Stone [5] which asserts that the classes of fully normal $T_{2}$ space and paracompact $T_{2}$ spaces coincide. We include this here as

LEMma 3.1. Every open covering of a fully normal $T_{2}$ space (=paracompact $T_{2}$ ) possesses a locally-finite star refinement.

For given an open covering $U$ of a fully normal space, there is a covering $V_{1}$ which star-refines $U$. By Stone's equivalence, $V_{1}$ has a locally-finite refinement which then star-refines the original covering.

4. The main theorem. Having defined the groups and the class of spaces of interest here, we may now state the principal theorem of this paper.

THEOREM 1. If $X$ is fully normal and $A$ is closed, then the homomorphism $\rho^{*}$ is an isomorphism onto.

Barring matters of technique, which here parallels Keesee, the essential idea is to modify a given cocycle slightly to produce one which 
is locally-finite valued and lies in the same coset $\bmod B^{p}(X, A)$. To establish this as well as the $1-1$ property in one construction, it will be convenient to proceed as follows.

Let $\mathcal{H}$ denote a collection of subsets of $G$ satisfying the conditions: (i) $M \in \mathscr{X} \cdot \rightarrow \cdot-M \in \mathcal{X}$, (ii) $M_{1}, M_{2} \in \mathcal{X} \cdot \rightarrow \cdot M_{1}+M_{2} \in \mathcal{X}$, and (iii) any nonvoid subset of a member of $\mathcal{X}$ is a member of $\mathcal{X}$. These conditions are satisfied if $\mathcal{X}$ consists of the zero subgroup only, or if $\mathscr{X}$ is the collection of all nonvoid finite subsets of $G$. For any such collection $\mathcal{X}$ define a subgroup $M^{p}(X) \subset C^{p}(X)$ as the collection of all $\phi \in C^{p}(X)$ such that there is an open covering $U$ of $X$ with $\phi\left(U^{p+1}\right)$ $\in \mathcal{X}$ for each $U \in \mathcal{U}$; further, let $M^{p}(X, A)=M^{p}(X) \cap C^{p}(X, A)$. With these definitions we observe that:

(4.1) If $\mathcal{X}$ consists of the subset $\{0\}$ only, then $M^{p}(X, A)=C_{0}^{p}(X)$, and

(4.2) If $\mathcal{X}$ consists of all finite subsets of $G$ and $X$ is paracompact, then

$$
M^{p}(X, A)=L^{p}(X, A)+C_{0}^{p}(X) .
$$

The first remark is obvious from the definition, and the only nontrivial part of (4.2) is the inclusion $M^{p}(X, A) \subset L^{p}(X, A)+C_{0}^{p}(X)$, which need only be established for $M^{p}(X)$, the general case following from an application of the Dedekind modular law. Therefore, if $\phi \in M^{p}(X)$, and since $X$ is paracompact, there is a locally-finite covering $U$ with $\phi\left(U^{p+1}\right) \in \mathcal{X}$ for each $U \in \mathcal{V}$. Defining $\psi: X^{p+1} \rightarrow G$ by $\psi|U(p+1)=\phi| U(p+1)$ and 0 elsewhere, we have that $\phi=\psi+(\phi-\psi)$ where clearly $\phi-\psi \in C_{0}^{p}(X)$. We assert that $\psi \in L^{p}(X)$, for given $\left(x_{0}, \cdots, x_{p}\right) \in X^{p+1}$, there is a neighborhood $P$ of $x_{0}$ for which $Q=\{U \in U \mid U \cap P$ is nonvoid $\}$ is finite. Letting $\pi$ denote the projection of $X^{p+1}$ onto its first coordinate, it follows easily that $\psi\left(\pi^{-1}(P)\right)$ is finite, for $\phi\left(\pi^{-1}(P) \cap \mho^{(p+1)}\right) \subset \phi\left(Q^{p+1}\right)$, which is finite.

The following lemma is a slight modification of one given by Keesee [3], which in turn generalizes the fundamental lemma used by Spanier [4].

Lemma 4.2. Suppose (a) $X$ and $Y$ are topological spaces and $f . g:(X, A) \rightarrow(Y, B)$ are functions on $(X, A)$ to $(Y, B)$ (not necessarily continuous);

(b) $\phi \in C^{p}(Y, B) \cap \delta^{-1} M^{p+1}(Y, B)$;

(c) $\mathcal{V}$ an open covering of $Y$ such that $\phi \mid \mathcal{V}(p+1) \cap B^{p+1}=0$, and $V \in \mathcal{V} \rightarrow \cdot \delta \phi\left(V^{p+2}\right) \in \mathcal{X}$;

(d) $U$ an open covering of $X$ such that, for each $U \in V$, there is a $V \in \mathcal{Y}$ such that $f(U) \cup g(U) \subset V$. 
Then, for $p>0, f^{\dagger} \phi-g^{\dagger} \phi \in \delta C^{p-1}(X, A)+M^{p}(X, A)$, and, for $p=0$, $f^{\dagger} \phi-g^{\dagger} \phi \in M^{0}(X, A)$.

Here, as in general, $f^{\sharp} \phi\left(x_{0}, \cdots, x_{p}\right)=\phi\left(f\left(x_{0}\right), \cdots, f\left(x_{p}\right)\right)$. The proof makes use of the cochain homotopy operator $D: C^{p+1}(Y)$ $\rightarrow C^{p}(X)$ defined by $D \phi\left(x_{0}, \cdots, x_{p}\right)=\sum_{i=0}^{p}(-1)^{i} \phi\left(g\left(x_{0}\right), \cdots\right.$, $\left.g\left(x_{i}\right), f\left(x_{i}\right), \cdots, f\left(x_{p}\right)\right)$ having the property for $p>0$ and $\phi \in C^{p}(Y)$ that $f^{\sharp} \phi-g^{\sharp} \phi=\delta D \phi-D \delta \phi$, and if $p=0$, then $f^{\sharp} \phi-g^{\sharp} \phi=D \delta \phi$. The proof is then completed by observing that for $p \geqq 0, D \delta \phi \in M^{p}(X, A)$ and, if $p>0, D \phi \in C^{p-1}(X, A)$, with the hypotheses as given. For details the reader is referred to the above cited proofs.

\section{LemMA 4.3. If $X$ is fully normal and $A$ is closed then}

$C^{p}(X, A) \cap \delta^{-1} M^{p+1}(X, A) \subset \delta C^{p-1}(X, A)+M^{p}(X, A)+L^{p}(X, A)$

if $p>0$, and

$$
C^{0}(X, A) \cap \delta^{-1} M^{1}(X, A) \subset M^{0}(X, A)+L^{0}(X, A) .
$$

Proof. If $\phi \in C^{p}(X, A) \cap \delta^{-1} M^{p+1}(X, A)$ then, since $A$ is closed, there is an open covering $\nu$ of $X$ such that (i) $\phi \mid \nu(p+1) \cap A^{p+1}=0$ and (ii) $V \in \mathcal{V} \rightarrow \cdot \delta \phi\left(V^{p+2}\right) \in \mathcal{X}$. By Lemma 3.1, there is a locallyfinite star refinement $V$ of $V$. Well order $U$ and for each nonvoid $U \in U$ select $x_{U} \in U$ (with $x_{U} \in U \cap A$ when this intersection is nonvoid). Define a function $f:(X, A) \rightarrow(Y, B)$ as follows: for each $x \in X$, $f(x)=x_{U}$ where $U$ is the first member of $U$ to which $x$ belongs. Clearly $f(A) \subset A$. Moreover, if $U \in U$, then $f(U) \subset$ st $(U) \subset V$ for some $V \in \mathcal{V}$. That is, for each $U \in \mathcal{V}$ there is a $V \in \mathcal{V}$ such that $f(U) \cup U \subset V$. With $(Y, B)=(X, A)$ and $g:(X, A) \rightarrow(X, A)$ the identity, the hypotheses of Lemma 4.2 are satisfied and therefore $f^{\sharp} \phi-\phi$ $\in C^{p-1}(X, A)+M^{p}(X, A)$ if $p>0$ and $f^{\star} \phi-\phi \in M^{0}(X, A)$ if $p=0$. Since $U$ is locally-finite, for each $x \in X$ there is an (open) neighborhood $P_{x}$ meeting only a finite number of members of $U$. Therefore if $\left(x_{0}, \cdots, x_{p}\right) \in X^{p+1}, P_{x_{0}} \times P_{x_{1}} \times \cdots \times P_{x_{p}}$ is open containing $\left(x_{0}, \cdots, x_{p}\right)$ and $f\left(P_{x_{0}}\right) \times \cdots \times f\left(P_{x_{p}}\right)$ is finite. Hence $f^{\sharp} \phi \in L^{p}(X)$. Moreover, since $\phi \mid \mathcal{V}^{(p+1)} \cap A^{p+1}=0$ it follows that $f^{\sharp} \phi \mid U^{(p+1)} \cap A^{p+1}=0$ and $f^{\sharp} \phi \in C^{p}(X, A)$. Therefore $f^{\sharp} \phi \in L^{p}(X, A)$ and $\phi \in \delta C^{p-1}(X, A)+M^{p}(X, A)+L^{p}(X, A)$, completing the proof.

We are now in a position to complete the proof of Theorem 1, observing first that, with the added hypotheses of remark (4.1) the conclusion of the above lemma reads

$$
C^{p}(X, A) \cap \delta^{-1} C_{0}^{p+1}(X) \subset \delta C^{p-1}(X, A)+C_{0}^{p}(X)+L_{0}^{p}(X, A),
$$

i.e., $Z^{p}(X, A) \subset B^{p}(X, A)+L^{p}(X, A)$. Therefore $Z^{p}(X, A)=B^{p}(X, A)$ $+Z_{L}^{p}(X, A)$ and $\rho^{*}$ is onto. 
To establish that $\rho^{*}$ is $1-1$ we must verify that $B_{L}^{p}(X, A)=B^{p}(X, A)$ $\cap Z_{L}^{p}(X, A)$. To this end select $\mathcal{X}$ as all finite subsets of $G$. Then, by the above lemma $\delta C^{p-1}(X, A) \cap M^{p}(X, A)=\delta\left[C^{p-1}(X, A) \cap \delta^{-1} M^{p}\right.$. $\cdot(X, A)] \subset \delta\left[\delta C^{p-2}(X, A)+M^{p-1}(X, A)+L^{p-1}(X, A)\right]$, and replacing $M^{p-1}(X, A)$ by $C_{0}^{p-1}(X)+L^{p-1}(X, A)$ we have $\delta C^{p-1}(X, A)$ $\cap M^{p}(X, A) \subset \delta C_{0}^{p-1}(X)+\delta L^{p-1}(X, A) \subset C_{0}^{p}(X)+\delta L^{p-1}(X, A)$. Hence, making free use of the Dedekind modular law,

$$
\begin{aligned}
B^{p}(X, A) \cap L^{p}(X, A) & =\left[\delta C^{p-1}(X, A)+C_{0}^{p}(X)\right] \cap M^{p}(X, A) \cap L^{p}(X, A) \\
& =\left[\delta C^{p-1}(X, A) \cap M^{p}(X, A)+C_{0}^{p}(X)\right] \cap L^{p}(X, A) \\
& \subset\left[\delta L^{p-1}(X, A)+C_{0}^{p}(X)\right] \cap L^{p}(X, A) \\
& =\delta L^{p-1}(X, A)+L_{0}^{p}(X)=B_{L}^{p}(X, A),
\end{aligned}
$$

from which the desired conclusion is immediate.

Corollary 1. If $X$ is compact $T_{2}$ and $A$ is closed then $H_{F}^{p}(X, A)$ $=H_{L}^{p}(X, A)$ and $\tau^{*}: H_{F}^{p}(X, A) \rightarrow H^{p}(X, A)$ is an isomorphism onto.

For in this case any cochain which is locally-finitely-valued is then finitely-valued and $F^{p}(X)=L^{p}(X)$. Moreover the homomorphism $\tau^{*}$ coincides with the isomorphism $\rho^{*}$, for $X$ is fully normal.

5. Cocycles with compact supports. Results analogous to the above may be obtained for the locally compact $T_{2}$ case if the cocycles are presumed to have compact supports. For a cochain $\phi \in C^{p}(X)$ we define $|\phi|=$ support of $\phi$ by $x \in|\phi|$ if and only if $\phi \mid P^{p+1} \neq 0$ for each neighborhood $P$ of $x$. It is well known that $|\phi|$ is a closed subset of $X$, that $|\delta \phi| \subset|\phi|$, and that the subgroup $C_{0}^{p}(X)$ consists exactly of those cochains with void supports. The main theorem has the following modification:

Theorem 2. If $X$ is locally compact $T_{2}$ and $A$ is closed, and if $\phi \in Z^{p}(X, A)$ has a compact support, then there is a finitely-valued cocycle, likewise with compact support, in the same equivalence class mod $B^{p}(X, A)$.

We begin the proof by first observing that we may assume that $\phi \mid X^{p+1} \backslash S^{p+1}=0$ for some compact subset $S$ of $X$. Since $|\phi|$ is compact and $X$ is locally compact $T_{2}$ there is a compact neighborhood $S$ of $|\phi|$. Defining $\phi^{\prime}$ to agree with $\phi$ on $S^{p+1}$ and to be zero otherwise, it follows easily that $\phi^{\prime} \in Z^{p}(X, A)$ and $\phi-\phi^{\prime} \in C_{0}^{p}(X) \subset B^{p}(X, A)$.

Hence, making the above substitution, let $U$ be an open covering of $X$ such that $\delta \phi \mid U^{(p+2)}=0$ and $\phi \mid U^{(p+1)} \cap A^{p+1}=0$. Since $S$ is compact it is covered finitely by $\left\{U_{1}, \cdots, U_{n}\right\}=V^{\prime} \subset U$. Let 
$\boldsymbol{V}=\left\{U_{1}, \cdots, U_{n}, X \backslash S\right\}$, a covering of $X$ for which $\delta \phi \mid \boldsymbol{V}^{(p+2)}=0$ and $\phi \mid \mathcal{V}^{(p+1)} \cap A^{p+1}=0$. Moreover, $\mathcal{V}^{\prime}=\left\{U_{i} \cap S\right\}_{i=1}^{n}$, as an open covering of $S$ (by sets open in $S$ ), possesses a finite star-refinement $\mathscr{W}^{\prime}$, for $S$ is compact normal. For each $W^{\prime} \in W^{\prime}$ the collection $\left\{U \in U^{\prime} \mid W^{\prime} \subset U \cap S\right\}$ is finite and hence $\bigcap\left\{U \in U^{\prime} \mid W^{\prime} \subset U \cap S\right\}$ is open containing $W^{\prime}$. Therefore there is a $W$ open in $X$ such that $W^{\prime}=W \cap S$ and $W \subset \cap\left\{U \in U^{\prime} \mid W^{\prime} \subset U \cap S\right\}$.

Let $\mathscr{W}=\left\{W \mid W^{\prime} \in \mathscr{W}^{\prime}\right\}$, a finite covering of $S$ by sets open in $X$. For each nonvoid $W_{i}^{\prime} \in \mathscr{W}^{\prime}$ select $x_{i} \in W_{i} \cap S=W_{i}^{\prime}$ (in $W_{i} \cap S \cap A$ if nonvoid). Define a function $f:(X, A) \rightarrow(X, A)$ by $f(x)=x$, if $x \in X \backslash S$, and $f(x)=x_{i}$ if $x \in W_{i}^{\prime}=W_{i} \cap S$ and $i$ is the first integer for which $x \in W_{i}$. Clearly, $f(A) \subset A$, and $f(X \backslash S)=X \backslash S$. Moreover, $f(W)$ $=f(W \cap S) \cup f(W \cap X \backslash S)$. Letting $W \cap S=W^{\prime}, f(W \cap S)=f(W)$ $\subset$ st $\left(W^{\prime}\right)$ which is contained in some $U \in U^{\prime}$. But, since $W^{\prime} \subset U$, by the above construction $W \subset U$ and we have verified that for each $W \in \mathscr{W}$ there is a $U \in \mathcal{V}^{\prime}$ such that $f(W) \cup W \subset U$. By Lemma 4.2, with $\mathcal{X}=\{0\}, f^{\sharp} \phi \in Z^{p}(X, A)$ and $f^{\ddagger} \phi-\phi \in B^{p}(X, A)$. It is clear from the construction that $f^{\prime}\left(S^{p+1}\right)$ is finite and $f^{\sharp} \phi \mid X^{p+1} \backslash S^{p+1}=0$. Combining the above statements $f^{\dagger} \phi$ is the desired finitely-valued cocycle.

\section{REFERENCES}

1. C. H. Dowker, Homology groups of relations, Ann. of Math. vol. 56 (1952) pp. $84-95$.

2. S. Eilenberg and N. Steenrod, Foundations of algebraic topology, Princeton, 1952.

3. J. W. Keesee, Finitely-valued cohomology groups, Proc. Amer. Math. Soc. vol. 1 (1950) pp. 418-422.

4. E. H. Spanier, Cohomology theory for general spaces, Ann. of Math. vol. 49 (1948) pp. 407-427.

5. A. H. Stone, Paracompactness and product spaces, Bull. Amer. Math. Soc. vol. 54 (1948) pp. 977-982.

6. J. W. Tukey, Convergence and uniformity in general topology, Annals of Mathematics Studies, no. 2, Princeton, 1940, p. 53.

7. C. T. Yang, On cohomology theories, Proc. Nat. Acad. Sci. U.S.A. vol. 38 (1952) pp. 348-351.

DUKE UNIVERSITY 